\title{
Study of Gribov copies in a Yang-Mills ensemble
}

\author{
D. Fiorentini $\odot,{ }^{1, *}$ D. R. Junior $\odot,{ }^{1,2, \dagger}$ L. E. Oxman $\odot,{ }^{1, \hbar}$ G. M. Simões, ${ }^{1, \S}$ and R. F. Sobreiro $\circledast^{1, \|}$ \\ ${ }^{1}$ UFF-Universidade Federal Fluminense, Instituto de Física, Campus da Praia Vermelha, \\ Avenida Litorânea s/n, 24210-346 Niterói, RJ, Brazil \\ ${ }^{2}$ University of Tübingen, Institut für Theoretische Physik, \\ Auf der Morgenstelle 14, D-72076 Tübingen, Germany
}

(Received 3 December 2020; accepted 10 May 2021; published 8 June 2021)

\begin{abstract}
Recently, based on a new procedure to quantize the theory in the continuum, it was argued that Singer's theorem points toward the existence of a Yang-Mills ensemble. In the new approach, the gauge fields are mapped into an auxiliary field space used to initially determine sectors labeled by center vortices, and then separately fix the gauge on them. In this work, we study this procedure in more detail. We provide examples of configurations belonging to these sectors and discuss the existence of non-Abelian degrees of freedom. Then, we discuss the importance of the mapping injectivity, and show that this property holds infinitesimally for typical configurations of the vortex-free sector and for the simplest example in the one-vortex sector. Finally, we show that these examples are free from Gribov copies.
\end{abstract}

DOI: 10.1103/PhysRevD.103.114010

\section{INTRODUCTION}

After Singer's theorem [1], it became clear that the usual Faddeev-Popov procedure to quantize non-Abelian YangMills theories must be somehow modified in the nonperturbative regime. Because of a topological obstruction, there is no condition $g(A)=0$ that can globally fix the gauge on the whole configuration space $\left\{A_{\mu}\right\}$. Hence, when such condition is imposed, the path integral still contains redundant degrees of freedom (d.o.f) associated with gauge fields obeying $g(A)=0$ and related by nontrivial gauge transformations. Such spurious configurations are typically called Gribov copies. The usual way to deal with this obstruction was implemented in the Landau gauge by V. N. Gribov in his seminal work [2], see also Ref. [3]. In his proposal, a path-integral restricted to a subset of $\left\{A_{\mu}\right\}$ was implemented so as to eliminate infinitesimal copies. As a consequence, the perturbative gauge propagator is destabilized, giving place to one with complex poles, while the ghost propagator is enhanced. Later on, many other developments were achieved. In the Landau gauge, D. Zwanziger was able to construct a local and renormalizable action [4]

\footnotetext{
*diego_fiorentini@id.uff.br

davidjunior@id.uff.br

leoxman@id.uff.br

§gustavomoreirasimoes@id.uff.br

"rodrigo_sobreiro@id.uff.br
}

Published by the American Physical Society under the terms of the Creative Commons Attribution 4.0 International license. Further distribution of this work must maintain attribution to the author(s) and the published article's title, journal citation, and DOI. Funded by SCOAP ${ }^{3}$. which was afterwards refined by the inclusion of dimension two condensates $[5,6]$. Beyond this gauge, it is worth mentioning important progress in the maximal Abelian gauge [7-9] and the linear covariant gauges [10-12], see also Ref. [13]. Finally, we refer to a Becchi-Rouet-StoraTyutin (BRST) invariant formulation of the path integral restriction, with a local and renormalizable action, that was implemented as a gauge independent recipe [14-16].

In Ref. [17], a different procedure to deal with Singer's obstruction was introduced, by splitting the configuration space into domains $\vartheta_{\alpha} \subset\left\{A_{\mu}\right\}$ where local sections are well defined. Of course, Singer's theorem does not pose any problem to define regions with a local section having no Gribov copies. The important point is that, in order for these regions to serve as a basis to implement the new proposal, they must form a partition

$$
\left\{A_{\mu}\right\}=\cup_{\alpha} \vartheta_{\alpha}, \quad \vartheta_{\alpha} \cap \vartheta_{\beta}=\varnothing \quad \text { if } \alpha \neq \beta .
$$

In that case, we would have $\left(S_{\mathrm{YM}}=\frac{1}{4 g^{2}} \int d^{4} x F_{\mu \nu}^{2}\right)$

$$
\begin{gathered}
Z_{\mathrm{YM}}=\sum_{\alpha} Z_{(\alpha)}, \quad\langle O\rangle_{\mathrm{YM}}=\sum_{\alpha} \frac{Z_{(\alpha)}}{Z_{\mathrm{YM}}}\langle O\rangle_{(\alpha)} \\
Z_{(\alpha)}=\int_{\vartheta_{\alpha}}\left[D A_{\mu}\right] e^{-S_{\mathrm{YM}}[A]}, \\
\langle O\rangle_{(\alpha)}=\frac{1}{Z_{(\alpha)}} \int_{\vartheta_{\alpha}}\left[D A_{\mu}\right] e^{-S_{\mathrm{YM}}[A]} O[A],
\end{gathered}
$$

and the usual Fadeev-Popov procedure could be separately implemented on each domain $\vartheta_{\alpha}$. In Ref. [17], motivated by 
lattice procedures used to detect center vortices by looking at the lowest eigenfunctions of the adjoint covariant Laplacian $[18,19]$, a partition of $\left\{A_{\mu}\right\}$ was generated in the continuum. For this purpose, we considered a gauge invariant auxiliary action $S_{\text {aux }}[A, \psi]$ for a tuple $\psi=$ $\left(\psi_{1}, \ldots, \psi_{N_{\mathrm{f}}}\right)$ of auxiliary adjoint scalar fields $\psi_{I}$, $I=1, \ldots, N_{\mathrm{f}}$. Then, the gauge field $A_{\mu}$ was correlated with the solution $\psi=\psi(A)$ to the set of classical equations of motion

$$
\frac{\delta S_{\mathrm{aux}}}{\delta \psi_{I}}=0, \quad \psi_{I} \in \mathfrak{g} \mathfrak{i t}(N), \quad I=1, \ldots, N_{\mathrm{f}},
$$

with appropriate boundary and regularity conditions. Since the auxiliary action is gauge invariant, when an orbit of $A_{\mu}$ is followed, an orbit in the auxiliary space $\{\psi\}$ is described, with components

$$
\begin{aligned}
\psi_{I}\left(A^{U}\right) & =U \psi_{I}(A) U^{-1}, \\
A_{\mu}^{U} & =U A_{\mu} U^{-1}+i U \partial_{\mu} U^{-1} .
\end{aligned}
$$

Next, a polar decomposition of the tuple $\psi$ was introduced. ${ }^{1}$

$$
\psi_{1}=S q_{1} S^{-1}, \ldots, \psi_{N_{\mathrm{f}}}=S q_{N_{\mathrm{f}}} S^{-1}
$$

based on a concept of "pure modulus" condition for a tuple $q=\left(q_{1}, \ldots, q_{N_{\mathrm{f}}}\right)$ :

$$
f(q)=0, \quad f \in \mathfrak{g} \mathfrak{i t}(N) .
$$

After this initial stage, we would have auxiliary variables $q(A)$ and $S(A)$ such that, when moving along the orbit of $A_{\mu}, q(A)$ stays invariant while the phase describes an orbit $S\left(A^{U}\right)=U S(A)$. The point is that even for smooth finiteaction configurations $A_{\mu}, S(A)$ will generally contain defects, which cannot be removed by means of the regular $U$-mappings associated with gauge transformations. That is, it is not possible to define a "unitary" gauge $S(A)=I$ on $\{A\}$. What can be done is to define regions $\mathcal{V}\left(S_{0}\right) \subset\left\{A_{\mu}\right\}$ formed by gauge fields that can be gauge-transformed to $S\left(A^{U}\right)=S_{0}$, where $U$ is regular and $S_{0}$ is a reference (class representative), characterized by a given distribution of defects. In other words, the gauge can be fixed with the $\mathcal{V}\left(S_{0}\right)$-dependent condition:

$$
\begin{aligned}
& f_{S_{0}}(A)=0, \quad A_{\mu} \in \mathcal{V}\left(S_{0}\right), \\
& f_{S_{0}}(A)=f\left(S_{0}^{-1} \psi_{1}(A) S_{0}, \ldots, S_{0}^{-1} \psi_{N_{\mathrm{f}}}(A) S_{0}\right) .
\end{aligned}
$$

As this is a local condition in the configuration space $\{A\}$, it is possibile to have no copies in this setting, while staying in line with Singer's theorem. Note also that any pair of

\footnotetext{
${ }^{1}$ In general, a relation between tuples of the form given in Eq. (6) shall be simply denoted by $\psi=q^{S}$.
}

different class representatives $S_{0}, S_{0}^{\prime}$ are such that $S_{0}^{\prime} \neq U S_{0}$ (for regular $U$ ) so that a gauge field $A_{\mu}$ cannot be in different regions. Then, as all the gauge fields belong to some region, the above procedure gives a partition of $\left\{A_{\mu}\right\}: \vartheta_{\alpha} \rightarrow \mathcal{V}\left(S_{0}\right)$. The labels correspond to oriented and nonoriented center vortices with non-Abelian degrees of freedom (d.o.f.), where the nonoriented component is generated by monopoles. Therefore, the YM field averages in Eq. (2) involve an ensemble integration over topological defects (sector labels) with a weight $Z_{\left(S_{0}\right)} / Z_{\mathrm{YM}}$ that is in principle calculable. Indeed, the all-orders perturbative renormalizability of the vortex-free sector was shown in Ref. [20]. The calculation of each sector, followed by the ensemble integration, is expected to give rise to the confining behavior in the nonperturbative regime. In this regard, these topological degrees have been established in the lattice as relevant for the confinement of quark probes in pure $S U(N)$ YM theory [21-31]. Moreover, a measure based on them has recently led to an effective model that captures the asymptotic properties of confinement [32]. Of course, this program tends to be prohibitively hard in the continuum. Nonetheless, understanding some of their facets could shed light on how to organize an approximation scheme on each sector. For example, in the calculation of quadratic fluctuations around a straight thin centervortex, different self-adjoint extensions are possible [33]. Which one to use should be determined from first principles and on physical grounds. This could also provide a guide to compute the different sectors in the lattice. The gauge-fixing method is based on many underlying assumptions. In this work, we aim at discussing them at the classical level, paying special attention to sectors that include center vortices. The purpose is to improve the understanding of the consistency of this procedure. In our proposal for continuum YM theories, this analysis was still lacking.

In Sec. II. we briefly review some well-known gaugefixings. Section III is devoted to compare them with our local procedure in the continuum, while in Sec. IV we clarify the necessary assumptions involved. Next, in Sec. V, we present examples of configurations that belong to sectors labeled by center vortices, and discuss the existence of non-Abelian d.o.f.. In Sec. VI we study the injectivity of the functional $\psi(A)$, and show that it is infinitesimally injective for typical configurations in the vortex-free sector, as well as for a particular configuration of the one-vortex sector. Finally, in Sec. VII, we establish the absence of Gribov copies for the configurations considered in Sec. VI.

\section{YANG-MILLS (GLOBAL) GAUGE-FIXINGS}

In this section, we provide a brief discussion of some of the gauge-fixings commonly used along the history of continuum and lattice non-Abelian gauge theories. These gauges are global, in the sense that a unique condition is 
imposed on the whole configuration space. This discussion will be useful to compare them with our local procedure and show, in the next section, how their problems and limitations could be avoided.

In the continuum, globally defined gauge-fixing conditions,

$$
f(A)=0, \quad A_{\mu} \in\left\{A_{\mu}\right\},
$$

were extensively studied. For example, the Landau gauge corresponds to $f(A)=\partial_{\mu} A_{\mu}$. Of course, due to Singer's (no-go) theorem [1], it is impossible to find a continuous condition on the whole configuration space $\left\{A_{\mu}\right\}$ such that $f\left(A^{U}\right)=0 \Rightarrow U=\mathbb{I}$. Then, in this framework, to continue working with the traditional methods, which are based on a single global $f(A)$, the path-integral was restricted to a subset of $\left\{A_{\mu}\right\}$. This is known as the first Gribov region, where infinitesimal copies are eliminated, although it generally contains finite copies. This region can also be defined as the smallest connected set, containing the trivial configuration $A_{\mu}=0$, such that the (gauge-dependent) Fadeev-Popov (FP) operator is positive definite [2]. In the infrared regime, it is believed that the YM path-integral in Landau gauge is dominated by the Gribov horizon [34,35], which is formed by configurations such that the corresponding FP operator has zero modes. These operators were studied in the continuum and in the lattice for the Coulomb and Landau gauges [36-38]. For example, in the Landau gauge, where the FP operator is given by

$$
M_{\text {Landau }}^{a b}=-\partial_{\mu} D_{\mu}^{a b} \delta^{(4)}(x-y),
$$

it was shown that smooth center vortices and instantons belong to the Gribov horizon [25,39,40].

In the lattice, center vortices and their properties have been extensively studied in the confining regime. In this case, although a gauge-fixing is not necessary to compute observables, it is relevant for the purpose of identifying the dominant configurations in the infrared regime. This was initially done within the Maximal Center Gauge (MCG) $[21,23,28]$, which brings each link element as close as possible to a center element. Given an initial field configuration $U_{\mu}(x) \in S U(N)$ (link-variables), the gauge is defined by the following maximization over gauge transformations $g(x)$

$$
\begin{aligned}
& \max _{g} \sum_{x, \mu}\left(\operatorname{tr} \operatorname{Ad}\left(U_{\mu}^{g}(x)\right)\right), \\
& \operatorname{Ad}\left(U_{\mu}^{g}(x)\right)=R^{\mathrm{T}}(x) \operatorname{Ad}\left(U_{\mu}(x)\right) R(x+\mu),
\end{aligned}
$$

where $R=\operatorname{Ad}(g)[\operatorname{Ad}(\cdot)$ denotes the adjoint representation of $S U(N)$ ]. In Ref. [28], this gauge was extended to the continuum by means of the requirement

$$
\min _{\Sigma} \min _{g} \int d^{D} x\left(\operatorname{tr}\left(A^{g}-a_{\Sigma}\right)^{2}\right)
$$

where $a_{\Sigma}$ is the gauge field for a thin vortex localized on $\partial \Sigma$, a closed surface. For local extrema, a condition can be obtained by first considering the minimization with respect to $G=e^{i \theta}$, with infinitesimal $\theta$, and fixed $\Sigma$ :

$$
\left[\partial_{\mu}+a_{\mu}^{\Sigma}, A_{\mu}\right]-\partial_{\mu} a_{\mu}^{\Sigma}=0 .
$$

If this step were free from Gribov copies, we would have a unique gauge field $A_{\Sigma}$ that satisfies Eq. (13), and the continuum maximal center gauge would be completed by determining the best $\Sigma$ :

$$
\min _{\Sigma} \int d^{D} x \operatorname{tr}\left(A\left[a_{\Sigma}\right]-a_{\Sigma}\right)^{2}
$$

On a conceptual level, this is an interesting procedure aimed at bringing $A_{\mu}$ as close as possible to a thin center vortex field $\left.a_{\Sigma}\right|_{\mu}$. However, as pointed out in Ref. [28], this route would require further improvements. This is due to the large mismatch between a smooth $A_{\mu}$ and a thin centervortex field $\left.a_{\Sigma}\right|_{\mu}$ at points that are close to any $\partial \Sigma$, where the difference $A-a_{\Sigma}$ is divergent. Thus, the condition (12) is always achieved for $a_{\Sigma}=0$, for vortexlike smooth configurations $A_{\mu}$. Among the possibilities to avoid this problem, a smoothed $a_{\Sigma}$ or the replacement $\operatorname{tr}(\cdot) \rightarrow s(\operatorname{tr}(\cdot))$ in Eq. (12), with $s(t)$ a monotonically increasing function, was considered in Ref. [28]. An issue pointed in that work is that, to avoid the divergence at $\partial \Sigma, s(t)$ cannot diverge as $t \rightarrow \infty$. However, this property would not penalize large deviations between $A_{\mu}$ and $\left.a_{\Sigma}\right|_{\mu}$ in other regions. In addition, for certain functions like $s(t)=-\tanh \left(R^{4} t^{2}\right)$, it was noted that the optimal $\partial \Sigma$ does not coincide with the guiding-center of a smooth center-vortex $A_{\mu}$, even for the simplest example.

Another important class of gauges in the lattice consider a set of eigenvectors $\phi^{(j)}$ corresponding to the lowest eigenvalues of the discretized covariant adjoint Laplacian,

$$
\Delta_{x y}^{a b}(U) \phi_{b}^{(j)}(y)=\mu_{j} \phi_{a}^{(j)}(x) .
$$

The gauge can then be fixed by imposing different conditions on the "lowest" eigenfunctions, i.e., the ones associated with the lowest eigenvalues. For instance, in the Laplacian center gauge (LCG) [18], the gauge is achieved by the composition of a pair of $S U(N)$ gauge transformations on the link variables. The first one is such that the lowest eigenfunction $\phi^{(1)}$ is oriented along the Cartan subalgebra. Then, a transformation is performed to make the color components of the second lowest eigenfunction $\phi^{(2)}$ satisfy some conventional conditions, while keeping $\phi^{(1)}$ fixed. The possibility of replacing the pair of Laplacian 
eigenfunctions by other adjoint fields in the continuum was first pointed out in Ref. [41], although a specific realization for these fields was not presented. In addition, the use of the above mentioned global gauge-fixing condition on these fields would lead to singular gauge-fixed fields, due to the large topological phases associated with center vortices. In the lattice, we would also like to mention the direct Laplacian center gauge (DLCG), introduced in Ref. [19] to address the above mentioned mismatch between smooth and thin configurations in the MCG. For $S U(2)$, instead of using the function $s(t)$, the smoothing of the MCG was done by promoting $R(x) \in S O(3)$ to a new degree of freedom $M(x)$, given by a $3 \times 3$ real matrix, and then performing the constrained maximization

$$
\begin{aligned}
& \max _{M} \sum_{x, \mu} \operatorname{tr}\left(M^{\mathrm{T}}(x) \operatorname{Ad}\left(U_{\mu}(x)\right) M(x+\mu)\right), \\
& \frac{1}{\mathcal{V}} \sum_{x} M^{\mathrm{T}}(x) M(x)=I_{3 \times 3},
\end{aligned}
$$

with $\mathcal{V}$ the lattice volume. Then, it was shown that the solution can be written in terms of the three lowest eigenfunctions of Eq. (15), $M_{a b}(x)=\phi_{b}^{(a)}(x)$. In the next step, an $S O(3)$-field was extracted from $M(x)$ through a polar decomposition. This field was then mapped to $S U(2)$ and the link-variables were gauge transformed to satisfy the adjoint version of the lattice Laplacian Landau Gauge (LLG) introduced in Ref. [42]. Finally, the DLCG was achieved by relaxing these link-variables to the closest configuration that satisfies the MCG. In Ref. [19], it was argued that the DLCG is preferable to the LLG since it avoids the presence of small scale fluctuations in the $\mathrm{P}$-vortex surfaces of projected configurations.

\section{THE LOCAL GAUGE-FIXING IN CONTINUUM YM THEORY}

In the lattice, the use of global gauge-fixing conditions, in the various center gauges discussed in Sec. II, is always possible because there is no concept of singular ill-defined phase field $S(x)$, when $x$ represents the discrete lattice sites. On the other hand, in the continuum, any attempt of defining a global condition, in a procedure that detects non-Abelian large topological phases $S(x), x \in \mathbb{R}^{4}$, would lead to singular gauge-fixed fields. For example, this occurs in the global gauge of Ref. [43]. In that case, among the natural large phases there are those corresponding to monopoles. Then, a gauge-fixing based on a global orientation of the auxiliary fields, where $S(x)$ is removed, leads to gauge fields $A_{\mu}$ containing singularities (Dirac strings). A similar situation would occur in gauge fixings in the continuum based on a set of adjoint auxiliary fields $\psi_{I} \in \mathfrak{g} \mathfrak{t}(N), \quad I=1, \ldots, N_{\mathrm{f}}$. This time, the topological phases $S(x) \in S U(N)$ will certainly include center-vortex defects. In addition, monopolelike phases will generally be attached to a pair of (physical) center-vortex defects. ${ }^{2}$ Again, there will be an obstruction to implement a global $\psi_{I}$ orientation, for every $A_{\mu} \in\left\{A_{\mu}\right\}$. By enforcing such a condition, ill-defined gauge fixed fields $A_{\mu}^{\mathrm{gf}}$ would be produced. On the other hand, in the continuum, it is precisely the clear distinction between regular and singular $S U(N)$-mappings that enables the introduction of the equivalence relation

$S(x) \sim S^{\prime}(x)$ if $\exists$ regular $U(x) / S^{\prime}(x)=U(x) S(x)$.

Such distinction and equivalence relation have no meaning for fields defined on the lattice. In the continuum, it enables us to think of generating, a priori, a catalog of different equivalence classes $\left[S_{0}\right]$, where $S_{0}(x)$ is a class representative. For example, in gauges based on adjoint auxiliary fields, a possible reference would be $S_{0}=e^{i \chi \beta \cdot T}$, where $\chi$ is a multivalued harmonic function and $\beta$ is a fundamental magnetic weight, such that $S_{0}$ changes by a center element when going around a closed surface $\partial \Sigma$. Of course, there is also a center-vortex free sector that can be labeled by $S_{0} \equiv I$. Other phases represent center-vortices that are nonoriented in the Lie algebra (see Refs. [28,32]). Here, we will not discuss the general classification of sectors. Instead, we shall analyze some examples. However, it is important to underline that, as noted in Ref. [32], multiplying a label $S_{0}$ by a regular mapping on the right generally leads to a physically inequivalent label. The identification of these non-Abelian degrees of freedom is an important property in the continuum which has no clear counterpart in the lattice. Using a mechanism that maps $A_{\mu}$ to $S$ in a gauge covariant way, we can look for the previously defined reference label $S_{0}$ that is equivalent to $S$. Then, instead of a global condition on $\left\{A_{\mu}\right\}$, we can require the gauge-fixed $A_{\mu}^{\mathrm{gf}}$ to be mapped into $S_{0}$, which is attained by a regular gauge transformation.

The simplest known example where local gauge-fixings are used is in the context of the Abelian Higgs Model [44]. In the unitary gauge, the phase of the Higgs field is required to be trivial. However, this condition cannot be applied to the Nielsen-Olesen vortex. For a straight infinite vortex, the best we can do is to fix the gauge field as $\phi=h e^{i \varphi}$, where $\varphi$ is the polar angle $\left(\partial^{2} \varphi=0\right)$. This is one of the motivations that led to the gauge-fixing proposal for pure YM theories in Ref. [17]. There, the construction of $S$ was done by introducing a set of adjoint auxiliary fields that minimize an auxiliary action

$$
S_{\text {aux }}=\int d^{4} x\left(\left\langle D_{\mu} \psi_{I}, D_{\mu} \psi_{I}\right\rangle+V_{\text {aux }}\right) .
$$

The Killing product is defined between elements of the Lie algebra according to

\footnotetext{
${ }^{2}$ These configurations are known as nonoriented center vortices (see Ref. [28]).
} 


$$
\langle X, Y\rangle=\operatorname{Tr}(\operatorname{Ad}(X) \operatorname{Ad}(Y)) .
$$

The consideration of $\psi(A)=\left(\psi_{1}, \ldots, \psi_{N_{\mathrm{f}}}\right)$, solution to this minimization problem [cf. Eq. (4)], has the advantage that, unlike the lowest eigenfunctions of the covariant Laplacian, it is a a well-posed problem in the continuum. Of course, at the quantum level, these fields were introduced by means of an identity, keeping the pure Yang-Mills dynamics unchanged. Regarding the field content and auxiliary potential, they were chosen such that the components $\psi_{I}$ of the classical solution $\psi(A)$ enable a simple concept of "modulus" tuple and the extraction of a phase. For this aim, we proposed $S_{\text {aux }}$ to display $S U(N) \rightarrow Z(N) \mathrm{SSB}$, which requires $N_{\mathrm{f}} \geq N$ (see also $\mathrm{Sec}$. IV). Among the many possible flavors of auxiliary fields, we preferred the choice $N_{\mathrm{f}}=N^{2}-1$, as a simple auxiliary action and procedure to extract the phase $S$ can be given for general $S U(N)$. For example, $V_{\text {aux }}$ can be chosen such that it is minimized by the nontrivial solutions to

$$
-i\left[\psi_{I}, \psi_{J}\right]=v f_{I J K} \psi_{K},
$$

namely, $\psi_{I}=v S T_{I} S^{-1}$, where $T_{I}, I=1, \ldots, N^{2}-1$ is the usual Lie basis. In regions where $A_{\mu}$ is close to a pure gauge, the solution will be close to a rotated frame. This "dynamical tendency" can be thought of as playing a similar role to the normalization and orthogonality property of the Laplacian eigenvector fields in the DLCG [cf. Eq. (16)].

The polar decomposition of a tuple $\psi$ [cf. Eqs. (6) and (7)] was done by defining a modulus tuple $q$ as the rotated $\psi$ that minimizes the average square distance

$$
\sum_{I}\left\langle q_{I}-v T_{I}\right\rangle^{2}
$$

This implies that $q_{I}$ is "aligned" with the Lie basis $T_{I}$ on average,

$$
\sum_{I}\left[q_{i}, T_{I}\right]=0
$$

Then, this procedure allows for the identification of the phase $S(A)$ of the solution $\psi(A)$ and identify the corresponding sector $\mathcal{V}\left(S_{0}\right)$ where $A_{\mu}$ is. Finally, the gauge can be fixed by the sector-dependent condition

$$
f_{S_{0}}(\psi)=\left[S_{0}^{-1} \psi_{I}(A) S_{0}, T_{I}\right]=0,
$$

see Eq. (8). This procedure, proposed in Ref. [17], has many points of contact with Laplacian center gauges used in the lattice. As discussed in Sec. II, the possibility of using adjoint fields other than the Laplacian eigenfunctions in the continuum was first pointed out in Ref. [41]. In our procedure we gave a realization of the auxiliary fields through a set of classical equations of motion while, instead of a pair, we considered various adjoint flavors. This field content simplified the extraction of a covariant phase out of $\psi$. Indeed, our concept of polar decomposition generalizes to $S U(N)$ the usual decomposition of the $3 \times 3$ real matrix, formed with the three lowest eigenvectors, used in the lattice adjoint LLG in $S U(2)$. In addition, as already explained, by considering local gauge-fixing conditions on $\mathcal{V}\left(S_{0}\right) \subset\left\{A_{\mu}\right\}$, we were able to avoid singular gaugefixed fields.

On the other hand, for oriented center vortices, our procedure differes from the continuum global MCG, as it is not based on comparing $A_{\mu}$ with the singular configurations $a_{\Sigma}$. The closed manifold $\partial \Sigma$ is not obtained after a best fit to $\left.a\right|_{\Sigma}$, but by reading the defects in $S(A)$. It is also very different from the traditional global gauge-fixings. For instance, in the Landau gauge, the Gribov copies associated with smooth center vortex or instanton configurations [cf. Eq. (10)] are related with zero mode solutions to a Schrödinger-like equation. It should be emphasized that the FP operator for this type of global gauges is completely different from the FP operator $J_{S_{0}}$ in any local sector $\mathcal{V}\left(S_{0}\right)$, which is related with the algebraic condition in Eq. (23). Therefore, there is no a priori reason to expect $J_{S_{0}}$ to contain zero modes. In order to address the possibility of copies, the analysis must be completely reformulated. Instead of considering a general $A_{\mu} \in\left\{A_{\mu}\right\}$, it should be separately done for $A_{\mu} \in \mathcal{V}\left(S_{0}\right)$, for every possible label $S_{0}$. As an initial step, to see if we are on a good direction, we will address some examples based on the simplest smooth center vortices. In particular, in Sec. V, we will show that the associated guiding-centers are correctly detected in the non-Abelian mapping $S(A)$, that the gauge-fixed field is regular, and that no copies will arise in this case.

\section{INVESTIGATING THE NEW PROCEDURE}

In the local procedure, if the solution to Eq. (4) is unique (after imposing regularity and boundary conditions), in a first step we may associate each field in $\left\{A_{\mu}\right\}$ with the auxiliary tuple $\psi(A)$ that minimizes the auxiliary action $S_{\text {aux }}[A, \psi]$. For this mapping to be useful to fix the gauge, a necessary condition is that different gauge fields of the same orbit are associated with different $\psi(A)$. If $\psi(A)$ is left invariant by nontrivial transformations $\psi_{I}(A) \rightarrow U \psi_{I}(A) U^{-1}$, $U \in S U(N)$, then no matter what the second step is, the final procedure will have gauge copies. If this is successful, in a second step, given a tuple $\psi(A)$, we would like to fix the gauge by imposing a condition that is satisfied by only one $\psi(A)$ as we move on the orbit of $A_{\mu}$. Again, because of Singer's theorem, it is impossible to find a global and continuous gauge-fixing condition. However, the idea is to implement a different gauge-fixing condition on each sector of a partition of $\left\{A_{\mu}\right\}$. In summary, for the gauge fixing to be well-defined, we need: 
1. Appropriate regularity and boundary conditions on the auxiliary fields so as to have a unique solution $\psi(A)$ to Eq. (4).

2. The auxiliary-field content and the auxiliary action must be such that $\psi(A)$ is injective on any gauge orbit. This means,

$$
\psi\left(A^{U}\right)=\psi(A) \Rightarrow U \in Z(N)
$$

where $Z(N)$ is the center group of $S U(N)$. This is just the requirement that the fields transform homogeneously under the gauge group in a LCG formulated in the continuum [41].

3. A univocally defined polar decomposition of $\psi(A)$. In this case, besides inducing the partition $\mathcal{V}\left(S_{0}\right)$, a local condition on $\mathcal{V}\left(S_{0}\right)$ with no copies,

$$
f_{S_{0}}(\psi(A))=0 \text { and } f_{S_{0}}\left(\psi\left(A^{U}\right)\right)=0 \Rightarrow U=\mathbb{I} \text {, }
$$

would be implemented in terms of the "pure modulus" concept discussed in Eqs. (7), (8) whose solution $^{3}$ is given by $\psi=q^{S_{0}}$.

If these requirements are fulfilled, we would have an $S_{0}$-dependent gauge-fixing condition, without copies on each local sector $\mathcal{V}\left(S_{0}\right)$ of the partition of $\left\{A_{\mu}\right\}$. As in other gauge-fixing procedures, the main idea is not to arrive at a closed expression for the gauge-fixed field $A_{\mu}^{\text {g.f. }}$ This could only be done for some specific cases. In fact, the objective is to properly quantize YM theory. Here, we shall briefly comment about the above requirements, relating them with the quantization procedure introduced in Ref. [17]. A detailed analysis will be developed in the next sections.

Regarding item 1, the natural regularity condition is to consider continuous single-valued auxiliary fields. In addition, as the gauge fields $A_{\mu}$ with finite YM action are asymptotically pure gauge, the natural boundary condition is that $\psi$ is covariantly constant at infinity,

$$
D_{\mu} \psi \rightarrow 0 \quad \text { when }|x| \rightarrow \infty
$$

This is consistent with the equations of motion if $\psi(x) \rightarrow$ $\bar{\psi}(x) \in \mathcal{M}$ in this limit, where $\mathcal{M}$ is the vacua manifold of $S_{\text {aux }}$. In Ref. [17], starting from the pure YM partition function,

$$
Z_{\mathrm{YM}}=\int\left[D A_{\mu}\right] e^{-S_{\mathrm{YM}}[A]},
$$

or the YM correlations, we introduced auxiliary fields satisfying Eq. (4) by means of an identity

\footnotetext{
${ }^{3}$ In general, a relation between tuples of the form given in Eq. (6) shall be simply denoted by $\psi=q^{S}$.
}

$$
1=\int[D \psi] \operatorname{det}\left(\frac{\delta^{2} S_{\mathrm{aux}}}{\delta \psi_{I} \delta \psi_{J}}\right) \delta\left(\frac{\delta S_{\mathrm{aux}}}{\delta \psi_{I}}\right),
$$

in the integrand of the $A_{\mu}$ path-integration. Given $A_{\mu}$, to correctly implement this identity, the argument of the $\delta$-functional must have a unique zero, and the quadratic operator in the determinant must be positive definite. This is nothing but the uniqueness requirement, which is met by the regularity and boundary conditions discussed above. In addition, the positivity of the quadratic form is related to solutions $\psi(A)$ with minimum auxiliary action.

Now, the manifold $\mathcal{M}$ must be nontrivial, that is, $S_{\text {aux }}$ must be constructed with an appropriate spontaneous symmetry breaking (SSB) pattern. If not, $\psi(A)$ could easily take values close to zero in a spacetime region, and the condition (24) in item 2 would be violated. In other words, we need $\psi(A)$ to be nontrivial almost everywhere to be able to extract information from it. Indeed, injectivity will be favored if points $\bar{\psi}$ in $\mathcal{M}$ satisfy (24), which corresponds to require an auxiliary action with an $S U(N) \rightarrow Z(N)$ spontaneous symmetry breaking (SSB) pattern. For this to happen, a minimum value of $N_{\mathrm{f}}=N$ flavors is needed (see Sec. IV). In this case, $\mathcal{M}=S U(N) / Z(N)=\operatorname{Ad}(S U(N))$, where $\operatorname{Ad}(\cdot)$ stands for the adjoint representation, and $S U(N)$ acts transitively on this manifold. Then, for a univocally defined polar decomposition (item 3), the asymptotic boundary condition would be

$$
\psi(x) \rightarrow \bar{\psi}(x)=u^{\bar{S}} \quad \text { when }|x| \rightarrow \infty,
$$

where $u$ is the pure modulus tuple in $\mathcal{M}$ and $\bar{S}=\bar{S}(x)$ is only defined at infinity by

$$
A_{\mu} \rightarrow \bar{S} \partial_{\mu} \bar{S}^{-1} \quad \text { when }|x| \rightarrow \infty
$$

Next, to represent the YM quantities in terms of a partition in the local sectors $\mathcal{V}\left(S_{0}\right)$, we introduced a second identity in the integrand of Eq. (28)

$$
\begin{aligned}
& 1=\sum_{S_{0}} 1_{S_{0}}, \quad 1_{S_{0}}=\int[D U] \delta\left(f_{S}(\psi)\right) \operatorname{det}(J(\psi)), \\
& S=U S_{0},
\end{aligned}
$$

where $J(q)$ is the Fadeev-Popov operator associated to the condition (25). According to item 3, the characteristic function $1_{S_{0}}$ is nontrivial on fields of the form $\psi=q^{S}$, $f(q)=0$. As $\psi$ is single-valued, when we get close to the defects of $S_{0}$, the fields accompanying Lie algebra components rotated by $S_{0}$ must tend to zero. When restricted to $\mathcal{V}\left(S_{0}\right)$, in order for the left-hand side of $1_{S_{0}}$ in Eq. (31) to be one, there should be a unique $U$ that solves $f_{S}(\psi)=0$. This is expected to be addressed by the consideration of the $S U(N) \rightarrow Z(N)$ SSB pattern and a good definition of polar 
decomposition with a univocally defined phase (and modulus).

Let us analyze some possibilities for the auxiliary action $S_{\text {aux }}$ in Eq. (18), initially focusing on the $S U(2)$ case. As $\operatorname{Ad}(S U(2))=S O(3)$, the group action on an adjoint scalar field can be pictured as an orthogonal rotation of a threecomponent vector. Then, noting that any vector is left invariant by an $S O(2)$ subgroup of rotations, we clearly see that it is not possible to produce $S U(2) \rightarrow Z(2) \mathrm{SSB}$ with a single scalar field. The situation is different if we consider two adjoint scalar fields

$$
S_{\mathrm{aux}}=\int d^{4} x\left(\left\langle D_{\mu} \psi_{1}, D_{\mu} \psi_{1}\right\rangle+\left\langle D_{\mu} \psi_{2}, D_{\mu} \psi_{2}\right\rangle+V\left(\psi_{1}, \psi_{2}\right)\right) .
$$

In this case, if the two vectors are linearly independent, it is clear that there will be no set of continuous transformations that leave them invariant. However, the potential must be chosen carefully. Asymptotically, the scalar fields should tend to $\mathcal{M}$. It is therefore important that we choose $V$ so as the field components of the tuples in $\mathcal{M}$ are linearly independent vectors. If we choose

$$
V\left(\psi_{1}, \psi_{2}\right)=\lambda_{1}\left(\left\langle\psi_{1}, \psi_{1}\right\rangle-v^{2}\right)^{2}+\lambda_{2}\left(\left\langle\psi_{2}, \psi_{2}\right\rangle-v^{2}\right)^{2},
$$

pathological configurations satisfying $\psi_{1}=\psi_{2}$, with $\left\langle\psi_{1}, \psi_{1}\right\rangle=v$, will belong to $\mathcal{M}$. They are left invariant by rotations with axis $\psi_{1}$. This can be fixed by adding the term $\left\langle\psi_{1}, \psi_{2}\right\rangle^{2}$ (see Ref. [45]. Then, $\mathcal{M}$ will consist of two orthogonal vectors which are only invariant by $Z(2) \subset S U(2)$ discrete transformations. Had we added $\left\langle\left[\psi_{1}, \psi_{2}\right],\left[\psi_{1}, \psi_{2}\right]\right\rangle$ instead, the pathology would persist. In general, among the interesting possibilities is the colorflavor symmetric action containing $N^{2}-1 S U(N)$ adjoint scalar fields $\psi_{I} \in \mathfrak{g} \mathfrak{u}(N), I=1, \ldots, N^{2}-1$, and auxiliary potential

$$
\begin{aligned}
V_{\mathrm{aux}}= & \frac{\mu^{2}}{2}\left\langle\psi_{I}, \psi_{I}\right\rangle+\frac{\kappa}{3} f_{I J K}\left\langle\psi_{I}, \psi_{J} \wedge \psi_{K}\right\rangle \\
& +\frac{\lambda}{4}\left(\left\langle\psi_{A} \wedge \psi_{B}\right\rangle\right)^{2} .
\end{aligned}
$$

where we introduced the notation $A \wedge B \equiv-i[A, B]$. In this case, the tuple $\psi(A)$ would be obtained by solving the equations

$$
D^{2}(A) \psi_{I}=\mu^{2} \psi_{I}+\kappa f^{I J K_{K}} \psi_{J} \wedge \psi_{K}+\lambda \psi_{J} \wedge\left(\psi_{I} \wedge \psi_{K}\right) .
$$

As argued in [46], this potential admits $S U(N) \rightarrow Z(N)$ $\mathrm{SSB}$, and is thus a good auxiliary action candidate (18) for general $S U(N)$. Indeed, $V_{\text {aux }}$ is minimized by tuples which are rotated Lie basis, satisfying Eq. (20), with

$$
v=-\frac{\kappa}{2 \lambda} \pm \sqrt{\left(\frac{\kappa}{2 \lambda}\right)^{2}-\frac{\mu^{2}}{\lambda}} .
$$

\section{PROPERTIES OF THE YANG-MILLS SECTORS}

In this section, we provide explicit examples of gauge field configurations belonging to nontrivial sectors labeled by center vortices. Then, we show that the procedure allows us to identify more general sectors labeled by non-Abelian d.o.f.. These are not related to ambiguities, but are in fact physically inequivalent possibilities located at the same center-vortex guiding centers.

\section{A. Some sectors labeled by a guiding center}

Let us start with some general remarks about thick center-vortex configurations of the form

$$
A_{\mu}=g a(x) \partial_{\mu} \chi \beta \cdot T,\left.\quad \beta \cdot T \equiv \beta\right|_{q} T_{q},
$$

where $\chi$ is a multivalued angle when we go around some closed surface $\Omega$ (guiding center), the elements $T_{q}$ $(q=1, \ldots, N-1)$ are Cartan generators of $\mathfrak{g} \mathfrak{u}(N)$, and $a(x)$ is a scalar profile that goes to 1 at infinity. In principle, this profile could be any smooth function. However, regularity conditions must be imposed on $a(x)$ to prevent singularities in $A_{\mu}$ and the associated $F_{\mu \nu}$. First of all, $a(x)=0$ at $\Omega$, otherwise $A_{\mu}$ would not be well defined there. Next, we evaluate

$F_{\mu \nu}=\left(\partial_{\mu} a \partial_{\nu} \chi-\partial_{\nu} a \partial_{\mu} \chi\right) \beta \cdot T+a(x)\left[\partial_{\mu}, \partial_{\nu}\right] \chi \beta \cdot T$.

We have $\left[\partial_{\mu}, \partial_{\nu}\right] \chi=0$ everywhere except at $\Omega$, where $a(x)=0$, so that we can disregard this term. When probing the behavior of $a(x)$ at points very close to $\Omega$, we can take $\chi=\varphi$, the angle of polar coordinates, with the $z-t$ plane taken as the tangent plane passing through the nearest point $x_{0} \in \Omega$. Consequently

$$
\begin{aligned}
\frac{1}{4}\left\langle F_{\mu \nu}, F^{\mu \nu}\right\rangle & =\frac{1}{2} \beta \cdot \beta\left(\partial_{\mu} a \partial^{\mu} a \partial_{\nu} \chi \partial^{\nu} \chi-\left(\partial_{\mu} a \partial^{\mu} \chi\right)^{2}\right) \\
& =\frac{1}{2 \rho^{2}} \beta \cdot \beta\left(\partial_{\mu} a \partial^{\mu} a-(\hat{\varphi} \cdot \nabla a)^{2}\right) .
\end{aligned}
$$

If we expand $a(x)=a^{(1)}(\varphi, z, t) \rho+a^{(2)}(\varphi, z, t) \rho^{2}+\ldots$, we must impose $a^{(1)}(\varphi, z, t)=0$ or, otherwise, the action would be infinite due to the divergence of $\left\langle F_{\mu \nu}, F^{\mu \nu}\right\rangle$ near $x_{0}$. In other words, on very general grounds, both $a(x)$ as well as its derivative in the local $\rho$ direction should vanish at every point of $\Omega$. In particular, this excludes thin-vortex configurations, as they are associated to an infinite YangMills action density. Thus, within our framework, typical 
calculations of the partial contribution $Z_{\left(S_{0}\right)}$ to the YangMills ensemble will consist of path-integrals with regularity conditions at the center-vortex guiding centers. This problem is similar to the computation of a Casimir energy, but with conditions imposed on surfaces with higher codimension $d=2$. In Ref. [47], the dynamical Casimir effect associated to a moving Dirichlet point was discussed for $d=1,2,3$. The case $d \geq 2$ was found more subtle to deal with, as it is necessary to renormalize the coupling to obtain a finite effective action for the particle. Codimension $d=2$ is particularly interesting as the coupling acquires dependence on an arbitrary mass scale $\mu$. In this case, it was found that the effective action contains a term proportional to $\dot{u}^{2}, u$ being an unitary tangent vector to the particle's trajectory. If we interpret the nontrivial trajectory of the particle as a curved vortexlike object, this term would be associated to stiffness. It would be interesting to generalize this calculation to gauge theories, This could allow to make contact with the observed properties of center vortices in the lattice, which display stiffness and tension terms $[32,48,49]$. It is also worth noting that investigations regarding quantum corrections to the effective action of a thin center-vortex were carried out in Ref. [33]. In particular, the one-loop correction to the thin vortex energy was shown to vanish for integer fluxes, for a particular choice of self-adjoint extension of the operator accompanying the fluctuations. The physical determination we are proposing here is different, so that the partial contribution of a center-vortex sector should be reexamined.

\section{B. Antisymmetric center vortices with charge $k$}

According to Eq. (31), if there is a regular transformation $U \in S U(N)$ such that

$$
\left[S_{0}^{-1} U^{-1}(x) \psi_{I}(x)(A) U(x) S_{0}, u_{I}\right]=0, \quad \forall x,
$$

where $u_{I}=v T_{I}$, then $A_{\mu} \in \mathcal{V}\left(S_{0}\right)$. In particular, $q_{I}(x)(A) \equiv$ $S_{0}^{-1} U^{-1}(x) \psi_{I}(x)(A) U(x) S_{0}$ must be single-valued. To proceed, we consider a class of configurations with cylindrical symmetry

$$
A_{\mu}^{(k)}=a_{(k)}(\rho) \partial_{\mu} \varphi \beta^{(k)} \cdot T,
$$

where $\rho, \varphi$ are the radial and angular coordinates (indices between parenthesis are not summed). The $(N-1)$-tuple $\beta^{k}$ is proportional to the weight of the k-antisymmetric representation: $\beta^{k}=2 N \sum_{i=1}^{k} \omega^{i}, k=1, \ldots, N-1$, where $\omega^{i}$ are the weights of the fundamental representation. The profile $a_{k}(\rho)$ satisfies the regularity conditions $a_{k}(\rho \rightarrow \infty)=1, \quad a_{k}(\rho=0)=0$. The first condition implies that these are in fact thick center-vortices, as they contribute a center element to the $k$ th power for large enough Wilson Loops that link them. In this case, $\psi(A)$ was obtained in Ref. [50], and is given by

$$
\begin{aligned}
& \psi_{q}^{(k)}=h_{q p}^{(k)} V_{(k)} T_{p} V_{(k)}^{-1}, \\
& \psi_{\alpha}^{(k)}=\psi_{\bar{\alpha}}^{(k)}=h_{\alpha}^{(k)} V_{(k)} T_{\alpha} V_{(k)}^{-1}, \\
& V_{(k)}=e^{i \varphi \beta^{k} \cdot T},
\end{aligned}
$$

where the profiles $a, h_{q p}, h_{\alpha}$ satisfy scalar equations, which were solved numerically for $S U(N)$. We now proceed to show that this solution satisfies Eq. (40), with $U=\mathbb{I}$. This implies that the gauge field already satisfies the gauge condition. It should be noted that, as expected, it is a regular configuration, even though the associated defect $V_{(k)}$ is not. As a first step, notice that $\left[\psi_{I}^{(k)}(x), u_{I}^{V_{(k)}}(x)\right]=$ $v \psi_{I J}^{(k)}(x) V_{(k)}(x)\left[T_{I}, T_{J}\right] V_{(k)}^{-1}(x)$. As $\psi_{I J}^{(k)}(x)$ is symmetric, this vanishes for all $x$. Finally, since $h_{\alpha}^{(k)}(0)=0$, for $\alpha \cdot \beta^{k} \neq 0$, the fields are single valued. Therefore, the configuration $A_{\mu}^{(k)}$ contributes to the sector $V_{0}^{(k)}$ for all values of the parameters consistent with SSB. Additionally, as the profiles $\psi_{I J}^{(k)}$ do not have other zeros, the configuration cannot contribute to a sector $V_{0}^{\prime}=e^{i \chi^{\prime} \beta^{\prime} \cdot T}$, if the guiding-center of $\chi^{\prime}$ is not located in the plane $\rho=0$. Moreover, for each $k$, the set of roots $\left\{\alpha_{r}\right\}$ that satisfy $\alpha$. $\beta^{k} \neq 0$ is different. To see this, consider, without loss of generality, that $k>k^{\prime}$. Then, for $p \leq k$ we have

$$
\begin{aligned}
\beta^{k} \cdot \alpha_{k^{\prime} p} & =-1, \\
\beta^{\left(k^{\prime}\right)} \cdot \alpha_{\left(k^{\prime}\right) p} & =0 .
\end{aligned}
$$

Since $h_{\alpha_{k^{\prime} p}}(0) \neq 0$, the solution $\psi_{I}^{\left(k^{\prime}\right)}$ will not contribute to the sector $V_{(k)}$, as it will not be single valued in the $\rho=0$ plane. Similarly, $\psi_{I}^{(k)}$ will not contribute to $V_{k^{\prime}}$, as for $q>k^{\prime}$

$$
\begin{gathered}
\beta^{k} \cdot \alpha_{k^{\prime} q}=0, \\
\beta^{\left(k^{\prime}\right)} \cdot \alpha_{\left(k^{\prime}\right) q}=1 .
\end{gathered}
$$

Therefore, the phases $V_{(k)}$ represent physically inequivalent center-vortex configurations that are located around the same points in spacetime (they have the same guidingcenters).

\section{Non-Abelian degrees of freedom}

In non-Abelian models with spontaneous symmetry breaking, vortices can have an internal orientational moduli [51-53]. In our case, although we are dealing with a pure gauge theory, a similar situation occurs when defining the different sectors. As discussed in Ref. [32], the multiplication of a general defect $S_{0}$ by a regular phase $\tilde{U}(x) \in$ $S U(N)$ on the right could yield a physically inequivalent label. For $S_{0}=e^{i \varphi \beta \cdot T}$, the new configuration is given by 


$$
\begin{aligned}
A_{\mu} & =a i S \partial_{\mu} S^{-1}=S \mathcal{A}_{\mu} S^{-1}+i S \partial_{\mu} S^{-1}, \\
\mathcal{A}_{\mu} & =(1-a) i S^{-1} \partial_{\mu} S \\
S & =\tilde{U} e^{i \varphi \beta \cdot T} \tilde{U}^{-1}
\end{aligned}
$$

while the associated solution can be written in the form $\psi_{A}=S \bar{\psi}_{A} S^{-1}$, where $\tilde{U}$ and $\bar{\psi}_{A}$ are single-valued and regular. Using

$$
\begin{gathered}
S^{-1} \partial_{\mu} S=\tilde{U} e^{-i \varphi \beta \cdot T} \tilde{U}^{-1} \partial_{\mu} \tilde{U} e^{i \varphi \beta \cdot T} \tilde{U}^{-1} \\
+i \partial_{\mu} \varphi \tilde{U} \beta \cdot T \tilde{U}^{-1}+\tilde{U} \partial_{\mu} \tilde{U}^{-1}, \\
S^{-1}\left(D_{\mu}(A) D^{\mu}(A) \psi_{A}\right) S= \\
\square \bar{\psi}_{A}+2 \mathcal{A}_{\mu} \wedge \partial_{\mu} \bar{\psi}_{A} \\
+\partial_{\mu} \mathcal{A}_{\mu} \wedge \bar{\psi}_{A} \\
+\mathcal{A}_{\mu} \wedge\left(\mathcal{A}_{\mu} \wedge \bar{\psi}_{A}\right),
\end{gathered}
$$

and the regularity conditions of $\bar{\psi}_{A}$ and $a(x)$ to expand $\bar{\psi}_{A}=\bar{\psi}_{A}^{(0)}+\bar{\psi}_{A}^{(1)} \rho+\ldots, \quad a(x)=a^{(1)} \rho+a^{(2)} \rho^{2}+\ldots$, we see that the term of order $\rho^{-2}$ in Eq. (51) is

$$
\begin{gathered}
\frac{\partial^{2} \bar{\psi}_{A}^{(0)}}{\partial \varphi^{2}}-2 \tilde{X} \wedge \frac{\partial \bar{\psi}_{A}^{(0)}}{\partial \varphi}+\tilde{X} \wedge\left(\tilde{X} \wedge \bar{\psi}_{A}^{(0)}\right), \\
\tilde{X}=\tilde{U} \beta \cdot T \tilde{U}^{-1}
\end{gathered}
$$

Since $\bar{\psi}_{A}$ is single-valued and regular, the zeroth order term $\bar{\psi}_{A}^{(0)}$ in the $\rho$-expansion cannot depend on $\varphi$. In addition, since the force $\frac{\delta S_{\text {aux }}}{\delta \psi_{A}}$ has no term of order $\rho^{-2}$, at the guiding center it must be verified

$$
\tilde{X} \wedge\left(\tilde{X} \wedge \bar{\psi}_{A}^{(0)}\right)=0 .
$$

Taking the scalar product with $\bar{\psi}_{A}^{(0)}$ and using the positivity of the metric, we get,

$$
\tilde{X} \wedge \bar{\psi}_{A}^{(0)}=0,
$$

which implies $\tilde{U}$-dependent regularity conditions on the components of $\bar{\psi}_{A}$ that do not commute with $\tilde{X}$. In this way, even when considering a $k=1$ fundamental center-vortex with a given guiding-center, we showed that there are gauge field configurations that belong to a continuum of physically inequivalent sectors of the Yang-Mills theory. These are genuine non-Abelian degrees of freedom that must be integrated in the YM ensemble.

\section{INFINITESIMAL INJECTIVITY OF $\psi(A)$}

In this section we shall see that injectivity is related to the positivity of the operator introduced in the identity of Eq. (28), and to the absence of nontrivial gauge transformations that leave invariant the auxiliary fields. Then, we show that the functional is injective for typical configurations of the vortex-free sector. A particular example in the one-vortex sector is also provided.

\section{A. Conditions for injectivity}

The equations of motion originated from the auxiliary action $\Sigma=\delta S / \delta \psi$ is a functional of $\psi$ and $A_{\mu}$, $S=S\left(\psi, A_{\mu}\right)$, and it is invariant under an infinitesimal gauge transformation, i.e., $\delta \Sigma=\delta_{A} \Sigma+\delta_{\psi} \Sigma=0$, with

$$
\delta_{A} \equiv \int \delta A_{\mu}^{a} \frac{\delta}{\delta A_{\mu}^{a}}, \quad \delta_{\psi} \equiv \int \delta \psi_{I}^{a} \frac{\delta}{\delta \psi_{I}^{a}} .
$$

Thus, by acting with a variation $\delta_{\psi}$ on $S$, we should get the corresponding solution to another gauge field on the same orbit, $A_{\mu}^{U}$. Then, we should study if

$$
\begin{aligned}
\delta_{A} \frac{\delta S}{\delta \psi_{I}^{a}(x)} & =-\delta_{\psi} \frac{\delta S}{\delta \psi_{I}^{a}(x)} \\
& =-\int d y \frac{\delta^{2} S}{\delta \psi_{I}^{a}(x) \psi_{J}^{b}(y)} f^{b m n} \xi^{m}(y) \psi_{J}^{n}(y) \\
& =0
\end{aligned}
$$

has nontrivial solutions. We may multiply this equation by $f^{a m^{\prime} n^{\prime}} \xi^{m^{\prime}}(x) \psi_{I}^{n^{\prime}}(x)$ and integrate over $x$ to arrive at

$$
\begin{aligned}
& \int d x d y \frac{\delta^{2} S}{\delta \psi_{I}^{a}(x) \psi_{J}^{b}(y)} v_{I}^{a}(x) v_{J}^{b}(y)=0, \\
& v_{I}^{a}(x)=f^{a m n} \xi^{m}(x) \psi_{I}^{n}(x)=\left.\left(\xi(x) \wedge \psi_{I}(x)\right)\right|_{a} .
\end{aligned}
$$

Since $\psi_{I}^{a}$ is a minimum of $S$, all the eigenvalues of $\frac{\delta^{2} S}{\delta \psi_{I}^{a}(x) \psi_{J}^{b}(y)}$ must be positive, as was already required for the identity in Eq. (28) to be well-defined. Therefore, nontrivial solutions for (7) are given by

$$
v_{I}^{a}=\delta \psi_{I}^{a}=0 .
$$

We see that the lack of injectivity is associated to the existence of nontrivial gauge transformations that leave $\psi_{I}$ invariant. By using the definitions $\Psi \equiv \psi_{A}^{B}, X \equiv \xi^{A} M^{A}$, $\left.M^{A}\right|_{B C} \equiv i f^{A B C}$, we can rewrite condition (58) for our choice of auxiliary action [Eq. (34)] as

$$
\Psi X=0 .
$$

For nontrivial gauge transformations, the solutions to Eq. (59) are related to the existence of zero-modes for $\Psi$. Therefore, we conclude that a lack of infinitesimal injectivity would be associated to configurations that satisfy $\operatorname{det} \Psi=0$. 


\section{B. Vortex-free sector}

For the vortex-free sector, in the limit of large $v$, we expect that $\Psi=v \mathbb{I}+\epsilon$, where $\epsilon$ is a small matrix. Defining $b(\epsilon)=\operatorname{det}(v \mathbb{I}+\epsilon)$, we must show that $b(\epsilon) \neq 0$ for small $\epsilon$. By expanding it, we may write

$$
b(\epsilon) \approx b(0)+\frac{\partial g}{\partial \epsilon^{a}} \epsilon^{a} .
$$

Since $b(0)=\operatorname{det} v \mathbb{I}=v^{N^{2}-1}$ is a finite (and large) value, we may conclude that the only solution to Eq. (59) in this regime is $X=0$. Hence, on the vortex-free sector, injectivity is ensured.

\section{Sectors with center-vortices}

The argument of the vortex-free sector cannot be extended to sectors labeled by vortices, as $\Psi$ will necessarily be far from the identity near their guiding-centers. We may, however, consider a particular example for $S U(2)$. The simplest case is the sector labeled by an antisymmetric vortex with charge $k=1$. Then, as $\beta=\sqrt{2}$, we have $S_{0}=e^{i \varphi \sqrt{2} T_{1}}$, where $\varphi$ is the angle of cylindrical

$$
\left(\begin{array}{c}
0 \\
-\xi_{3} h(\rho) \cos (\varphi)-\xi_{2} h(\rho) \sin (\varphi) \\
-\xi_{3} h(\rho) \cos (\varphi)+\xi_{2} h(\rho) \sin (\varphi)
\end{array}\right.
$$

For $\rho \neq 0$, this gives $\xi_{1}=\xi_{2}=\xi_{3}=0$. The only problematic region is the plane $\rho=0$, which is a region of null measure in $R^{4}$. The gauge transformations that would leave $\Psi$ invariant, thus leading to the lack of injectivity, should be different from the identity only in this plane. Such transformations are not continuous, so they can be disregarded. The functional $\psi(A)$ is therefore infinitesimally injective in the one-vortex sector for this particular example.

\section{A POLAR DECOMPOSITION WITHOUT INFINITESIMAL COPIES}

As discussed in Sec. IV, the injectivity of $\psi(A)$ does not guarantee that the gauge-fixing is free from copies. We still need to show that, for all sectors $S_{0}$,

$$
f_{S_{0}}(\psi(A))=f_{S_{0}}\left(\psi\left(A^{U}\right)\right)=0 \rightarrow U=\mathbb{I} .
$$

We shall see that this condition is related to the absence of zero modes of the operator introduced in the identity of Eq. (31). For instance, to analyze Eq. (65) in the vortex-free sector, we must show that if

$$
\left.\left(q_{I} \wedge T_{I}\right)\right|_{\gamma}=f^{a I \gamma} q_{I}^{a}=0,
$$

coordinates. For $S U(2)$, the solution $\psi(A)$, when $A$ is a minimum of the action as well, is known to be [54]

$$
\begin{aligned}
\psi_{1} & =h_{1}(\rho) T_{1}, \\
\psi_{\alpha_{1}} & =h(\rho) S_{0} T_{\alpha_{1}} S_{0}^{-1}, \\
\psi_{\overline{\alpha_{1}}} & =h(\rho) S_{0} T_{\overline{\alpha_{1}}} S_{0}^{-1} .
\end{aligned}
$$

In this case, there is only one root $\alpha_{1}=\frac{1}{\sqrt{2}}$, satisfying $\alpha_{1} \cdot \beta=1$, and the following relations hold

$$
\begin{aligned}
& S_{0} T_{\alpha_{1}} S_{0}^{-1}=\cos (\varphi) T_{\alpha_{1}}-\sin (\varphi) T_{\overline{\alpha_{1}}}, \\
& S_{0} T_{\overline{\alpha_{1}}} S_{0}^{-1}=\cos (\varphi) T_{\alpha_{1}}+\sin (\varphi) T_{\overline{\alpha_{1}}} .
\end{aligned}
$$

This implies the following $\Psi$ matrix:

$$
\left(\begin{array}{ccc}
h_{1}(\rho) & 0 & 0 \\
0 & h(\rho) \cos (\varphi) & -h(\rho) \sin (\varphi) \\
0 & h(\rho) \cos (\varphi) & h(\rho) \sin (\varphi)
\end{array}\right)
$$

Now, the condition (59) implies

$\left.\begin{array}{cc}h_{1}(\rho) \xi_{3} & h_{1}(\rho) \xi_{2} \\ \xi_{1} h(\rho) \sin (\varphi) & \xi_{1} h(\rho) \cos (\varphi) \\ -\xi_{1} h(\rho) \sin (\varphi) & \xi_{1} h(\rho) \cos (\varphi)\end{array}\right)=0$.

then there is no gauge transformation with nontrivial parameters $\xi^{a}$, such that

$$
f^{a I \gamma} f^{a n m} q_{I}^{n} \xi^{m}=0
$$

Of course these are just necessary conditions that a problematic tuple should satisfy, as $q_{I}$ should also minimize the auxiliary action (34). These algebraic conditions (66), (67) can also be written by using the generators in the adjoint representation:

$$
\left.\left.\operatorname{Ad}\left(T_{A}\right)\right|_{B C} \equiv M_{A}\right|_{B C}=i f^{A B C}
$$

and of the matrix

$$
\left.Q\right|_{I a}=q_{I}^{a} .
$$

Then, Eqs. (66) and (67) become, respectively,

$$
\begin{aligned}
\operatorname{Tr}\left(M_{b} Q\right) & =0, \\
\operatorname{Tr}\left(M_{\gamma} M_{b} Q\right) \xi^{\gamma} & =0 .
\end{aligned}
$$

We may write these conditions as 


$$
J^{A B} \xi^{B}=0, \quad J^{A B} \equiv \operatorname{Tr}\left(M^{A} M^{B} Q\right),
$$

and conclude that copies are associated with configurations having $\operatorname{det} J=0$. In fact, in Ref. [17], the operator $J$ is introduced in the Yang-Mills partition function by means of the Fadeev-Popov procedure [see Eq. (31)]. It is therefore expected that copies are related to zeros of this determinant.

Let us start by analyzing the above equations for $S U(2)$. In this case, $f^{A B C}=\frac{\epsilon^{A B C}}{\sqrt{2}}$, and the matrices $M$ and $X$ thus read

$$
\begin{aligned}
M_{1} & =\left(\begin{array}{ccc}
0 & 0 & 0 \\
0 & 0 & \frac{i}{\sqrt{2}} \\
0 & -\frac{i}{\sqrt{2}} & 0
\end{array}\right), \quad M_{2}=\left(\begin{array}{ccc}
0 & 0 & -\frac{i}{\sqrt{2}} \\
0 & 0 & 0 \\
\frac{i}{\sqrt{2}} & 0 & 0
\end{array}\right) \\
M_{3} & =\left(\begin{array}{ccc}
0 & \frac{i}{\sqrt{2}} & 0 \\
-\frac{i}{\sqrt{2}} & 0 & 0 \\
0 & 0 & 0
\end{array}\right), \\
X & =\xi^{A} M^{A}=\left(\begin{array}{ccc}
0 & \frac{i}{\sqrt{2}} \xi_{3} & -\frac{i}{\sqrt{2}} \xi_{2} \\
-\frac{i}{\sqrt{2}} \xi_{3} & 0 & \frac{i}{\sqrt{2}} \xi_{1} \\
\frac{i}{\sqrt{2}} \xi_{2} & -\frac{i}{\sqrt{2}} \xi_{1} & 0
\end{array}\right)
\end{aligned}
$$

The pure modulus condition (70) implies that $Q$ is a symmetric matrix, and thus can be parametrized as

$$
Q=\left(\begin{array}{lll}
Q_{11} & Q_{12} & Q_{13} \\
Q_{12} & Q_{22} & Q_{23} \\
Q_{13} & Q_{23} & Q_{33}
\end{array}\right) .
$$

The equation for copies (71) then reads

$$
\begin{aligned}
J^{a b} \xi^{b} & =0, \\
J & =\left(\begin{array}{ccc}
Q_{22}+Q_{33} & -Q_{12} & -Q_{13} \\
-Q_{12} & Q_{11}+Q_{33} & -Q_{23} \\
-Q_{13} & -Q_{23} & Q_{11}+Q_{22}
\end{array}\right), \\
\xi & =\left(\begin{array}{c}
\xi^{1} \\
\xi^{2} \\
\xi^{3}
\end{array}\right) .
\end{aligned}
$$

In order for the system (75) to have a nontrivial solution, the determinant of $C$ should be 0 (this is a necessary condition). This yields

$$
\begin{aligned}
\operatorname{det} J= & \left(Q_{22}+Q_{33}\right)\left(Q_{11}+Q_{33}\right)\left(Q_{11}+Q_{22}\right) \\
& -2 Q_{12} Q_{23} Q_{13}-Q_{12}^{2}\left(Q_{11}+Q_{22}\right)-Q_{23}^{2}\left(Q_{22}+Q_{33}\right) \\
& -Q_{13}^{2}\left(Q_{11}+Q_{33}\right)=0 .
\end{aligned}
$$

\section{A. Study of copies in the vortex-free sector}

In the vortex-free sector, for the general group $S U(N)$, the gauge-fixed functional $q_{I}(A)$ satisfies

$$
\begin{gathered}
q_{I}(A) \wedge u_{I}=0, \\
q_{I}(A) \rightarrow v T_{I}, \quad x \rightarrow \infty .
\end{gathered}
$$

If there is a copy, then there exists a gauge transformation $U(x)$ such that

$$
\begin{gathered}
q_{I}^{U}(A) \wedge u_{I}=0, \\
U(x) \rightarrow \mathbb{I}, \quad x \rightarrow \infty .
\end{gathered}
$$

For infinitesimal transformations, Eq. (80) reads

$$
f^{a I \gamma} f^{a n m} q_{I}^{n} \xi^{m}=0
$$

In the vortex-free sector, the boundary condition of Eq. (78) will imply (on the limit of large $v$ ) that the fields $\mathrm{Q}$ are close to $v \mathbb{I}$ everywhere, i.e., $q_{I}^{a}=\delta_{I}^{a}+\epsilon_{I}^{a}$. Equation (81) thus becomes

$$
\begin{aligned}
\xi^{\gamma}+f^{a I \gamma} f^{a n m} \xi^{n} \epsilon_{I}^{m} & =0, \\
\xi^{m}\left(\delta^{m \gamma}+f^{a I \gamma} f^{a n m} \epsilon_{I}^{n}\right) & =0 .
\end{aligned}
$$

This yields a system of $N^{2}-1$ linear equations in the variables $\xi^{a}$, with coefficients that will depend on $\epsilon_{I}^{a}$, i.e.,

$$
M(\epsilon) \xi=0,
$$

where $M$ is the matrix of coefficients. For this system to have a nontrivial solution, a necessary condition is

$$
k(\epsilon) \equiv \operatorname{det} M(\epsilon)=0 .
$$

Since $k(\epsilon)$ is polynomial on the infinitesimal parameters $\epsilon_{I}^{a}$, we may approximate:

$$
k(\epsilon) \approx k(0)+\frac{\partial k(\epsilon)}{\partial \epsilon_{I}^{a}} \epsilon_{I}^{a} .
$$

As $M(0)$ is simply the $\left(N^{2}-1\right) \times\left(N^{2}-1\right)$ identity matrix, we have $k(0)=1$, a finite value. Therefore, in the large $v$-limit, there are no Gribov copies for the dominant configurations in the vortex-free sector.

\section{B. Study of copies in a general sector}

In a general sector labeled by a defect $S_{0}$, the functional $\psi_{I}(A)$ satisfies 


$$
\frac{\delta S_{\mathrm{aux}}}{\delta \psi_{I}}=0
$$

For a general $A$ in this sector, $\psi$ will be of the form $\psi_{I}=U S_{0} q_{I} S_{0}^{-1} U^{-1}$, with $U$ regular. The gauge-fixed $A_{\mu}$ will be associated to $\zeta_{I} \equiv S_{0} q_{I} S_{0}^{-1}$, and should satisfy

$$
\begin{gathered}
\zeta_{I}(A) \wedge \eta_{I}=0, \\
\eta_{I} \equiv v S_{0} T_{I} S_{0}^{-1}, \\
\zeta_{I}(A) \rightarrow v S_{0} T_{I} S_{0}^{-1}, \quad x \rightarrow \infty .
\end{gathered}
$$

If there is a copy, then there exists a gauge transformation $U(x)$ such that

$$
\begin{gathered}
\zeta_{I}^{U}(A) \wedge \eta_{I}=\left(U S_{0} q_{I} S_{0}^{-1} U^{-1}\right) \wedge S_{0} T_{I} S_{0}^{-1}=0, \\
U(x) \rightarrow \mathbb{I}, \quad x \rightarrow \infty .
\end{gathered}
$$

We may write condition (91) in terms of $q_{I}$ :

$$
\left(S_{0}^{-1} U S_{0} q_{I}\left(S_{0}^{-1} U S_{0}\right)^{-1}\right) \wedge u_{I}=0 .
$$

In terms of the matrix $Q$ defined in the previous section, this is

$$
R\left(S_{0}^{-1} U S_{0}\right) Q=Q^{\prime}
$$

with $Q, Q^{\prime}$ being pure modulus matrices. By defining $\tilde{U} \equiv S_{0}^{-1} U S_{0}$, we arrive at the conditions that problematic matrices $Q$ should satisfy:

$$
\begin{gathered}
R(\tilde{U}) Q=Q^{\prime}, \\
\tilde{U}(x) \rightarrow \mathbb{I}, \quad x \rightarrow \infty .
\end{gathered}
$$

An important fact that follows from the definition of $\tilde{U}$ is that if $U$ is infinitesimal, so is $\tilde{U}$. This is so because $S_{0} \in S U(N)$, so that it preserves the norm of the vector $\xi$. Specifically,

$$
\begin{aligned}
U & =\mathbb{I}+\xi^{A} T^{A} \rightarrow \tilde{U}=\mathbb{I}+\left(\xi^{\prime}\right)^{A} T^{A}, \\
\xi^{\prime} & =R\left(S_{0}\right) \xi .
\end{aligned}
$$

The equation for infinitesimal copies is therefore the same in all sectors. However, in a general sector there is no reason to believe that $q_{I}$ will be close to $v T_{I}$ everywhere, since some of its components must go to zero at the guiding centers of the vortices. Gauge transformations with parameters that are nonzero only in these regions surrounding the guiding-centers of the vortices could, in principle, yield copies. However, as $v$ grows, these regions become increasingly small.
An example of configuration that could yield copies is when $A_{\mu}=a(\rho) \partial_{\mu} \varphi \beta \cdot T$, belonging to the sector labeled by a vortex along the $\mathrm{z}$ axis. As discussed in (61), for $S U(2)$, the solution for $\psi(A)$ is known. It is of the form

$$
\psi_{I}=h_{I J} S_{0} T_{I} S_{0}^{-1} .
$$

This implies

$$
q_{I}=h_{I J} T_{J}
$$

The associated $Q$-matrix is symmetric, as required by the gauge fixing. For this to admit infinitesimal copies, Eq. (76) should be satisfied at some finite region. The necessary condition for the existence of copies is [Eq. (76)]

$$
2 h\left(h_{1}+h\right)^{2}=0 .
$$

Since the profiles $h_{1}(\rho)$ and $h(\rho)$ are positive for all $\rho>0$ (see Ref. [54]), it is easy to see that this condition is only satisfied at $\rho=0$, which is a region in $R^{4}$ of null measure. The transformations that lead to copies are not continuous, as they should be nontrivial only in this plane. Then, they should not be considered as associated to gauge transformations. This configuration, therefore, does not admit Gribov copies.

\section{CONCLUSIONS}

In this work, we studied the consistency of a recently proposed procedure to fix the gauge on different sectors of the gauge-field configuration space $\left\{A_{\mu}\right\}$. Unlike the usual procedure, based on a unique gauge-fixing condition and a restriction to the first Gribov region (to avoid infinitesimal copies), our proposal is based on the consideration of different local conditions on the infinitely many sectors of a partition of $\left\{A_{\mu}\right\}$. These sectors are labeled by oriented and nonoriented center vortices, and the Yang-Mills path-integral measure includes a sum over partial contributions. Our procedure is suited to detect the microscopic features of center vortices in the continuum, which in global gaugefixing conditions, like the Landau gauge, are effectively seen signaling the breaking of the perturbative regime at the Gribov horizon [40,55]. Each partial contribution can be associated to a problem written in a form closer to the usual one. Here, along the way, we clarified the relevance of the regularity conditions to solve the auxiliary field equations and provide a physical determination of center-vortex sectors. In principle, this is different from considering a thin or thick center-vortex background plus quantum fluctuations. Instead, it is based on path integrating over gauge and auxiliary fields with given singular phases and regularity conditions. We provided explicit examples of thick centervortex configurations belonging to nontrivial sectors. We also discussed the existence of non-Abelian degrees of freedom, which are related to physically inequivalent labels 
with the same guiding centers. Finally, we showed the absence of Gribov copies for typical configurations of the vortex-free sector and for the simplest example in the sector labelled by a center vortex. This points to the idea that a possibility to deal with the Singer's obstruction to a global gauge-fixing is to approach Yang-Mills theories as an ensemble of center-vortex degrees.

In a future work, it would be interesting to establish the absence of copies for more general configurations of the oriented and nonoriented center-vortex sectors, and for more general values of the gauge-fixing parameters. This result, together with the all-orders perturbative renormalizability of these sectors, are important steps toward the establishment of the Yang-Mills ensemble in the continuum.

\section{ACKNOWLEDGMENTS}

The Conselho Nacional de Desenvolvimento Científico e Tecnológico (CNPq), the Coordenação de Aperfeiçoamento de Pessoal de Nível Superior (CAPES) - Finance Code 001, the Fundação de Amparo à Pesquisa do Estado do Rio de Janeiro (FAPERJ), and the Deutscher Akademischer Austauschdienst (DAAD) are acknowledged for their financial support.
[1] I. M. Singer, Some remarks on the Gribov ambiguity, Commun. Math. Phys. 60, 7 (1978).

[2] V. N. Gribov, Quantization of non-Abelian gauge theories, Nucl. Phys. B139, 1 (1978).

[3] R. F. Sobreiro and S. P. Sorella, Introduction to the Gribov ambiguities in Euclidean Yang-Mills theories, in 13th Jorge Andre Swieca Summer School on Particle and Fields Campos do Jordao, Brazil, 2005 (2005), arXiv:hep-th/ 0504095 .

[4] D. Zwanziger, Local and renormalizable action from the Gribov horizon, Nucl. Phys. B323, 513 (1989).

[5] D. Dudal, J. A. Gracey, S. P. Sorella, N. Vandersickel, and H. Verschelde, A refinement of the Gribov-Zwanziger approach in the Landau gauge: Infrared propagators in harmony with the lattice results, Phys. Rev. D 78, 065047 (2008).

[6] D. Dudal, S. P. Sorella, and N. Vandersickel, The dynamical origin of the refinement of the Gribov-Zwanziger theory, Phys. Rev. D 84, 065039 (2011).

[7] M. A. L. Capri, V. E. R. Lemes, R. F. Sobreiro, S. P. Sorella, and R. Thibes, The influence of the Gribov copies on the gluon and ghost propagators in Euclidean Yang-Mills theory in the maximal Abelian gauge, Phys. Rev. D 72, 085021 (2005).

[8] M. A. L. Capri, V. E. R. Lemes, R. F. Sobreiro, S. P. Sorella, and R. Thibes, A study of the maximal Abelian gauge in SU(2) Euclidean Yang-Mills theory in the presence of the Gribov horizon, Phys. Rev. D 74, 105007 (2006).

[9] M. A. L. Capri, A. J. Gomez, M. S. Guimaraes, V. E. R. Lemes, and S. P. Sorella, Study of the properties of the Gribov region in SU(N) Euclidean Yang-Mills theories in the maximal Abelian gauge, J. Phys. A 43, 245402 (2010).

[10] R. F. Sobreiro and S. P. Sorella, A study of the Gribov copies in linear covariant gauges in Euclidean Yang-Mills theories, J. High Energy Phys. 06 (2005) 054.

[11] M. A. L. Capri, A. D. Pereira, R. F. Sobreiro, and S. P. Sorella, Non-perturbative treatment of the linear covariant gauges by taking into account the Gribov copies, Eur. Phys. J. C 75, 479 (2015).
[12] M. A. L. Capri, D. Fiorentini, A. D. Pereira, R. F. Sobreiro, S. P. Sorella, and R. C. Terin, Aspects of the refined GribovZwanziger action in linear covariant gauges, Ann. Phys. (Amsterdam) 376, 40 (2017).

[13] A. D. Pereira and R. F. Sobreiro, On the elimination of infinitesimal Gribov ambiguities in non-Abelian gauge theories, Eur. Phys. J. C 73, 2584 (2013).

[14] M. A. L. Capri, D. Dudal, D. Fiorentini, M. S. Guimaraes, I. F. Justo, A. D. Pereira, B. W. Mintz, L. F. Palhares, R. F. Sobreiro, and S. P. Sorella, Exact nilpotent nonperturbative BRST symmetry for the Gribov-Zwanziger action in the linear covariant gauge, Phys. Rev. D 92, 045039 (2015).

[15] M. A. L. Capri, D. Dudal, D. Fiorentini, M. S. Guimaraes, I. F. Justo, A. D. Pereira, B. W. Mintz, L. F. Palhares, R. F. Sobreiro, and S. P. Sorella, Local and BRST-invariant YangMills theory within the Gribov horizon, Phys. Rev. D 94, 025035 (2016).

[16] M. A. L. Capri, D. Fiorentini, and S. P. Sorella, Gribov horizon and non-perturbative BRST symmetry in the maximal Abelian gauge, Phys. Lett. B 751, 262 (2015).

[17] L. E. Oxman and G. C. Santos-Rosa, Detecting topological sectors in continuum Yang-Mills theory and the fate of BRST symmetry, Phys. Rev. D 92, 125025 (2015).

[18] Ph. de Forcrand and M. Pepe, Center vortices and monopoles without lattice Gribov copies, Nucl. Phys. B598, 557 (2001).

[19] M. Faber, J. Greensite, and S. Olejník, Direct Laplacian center gauge, J. High Energy Phys. 01 (2001) 053.

[20] D. Fiorentini, D. R. Junior, L. E. Oxman, and R. F. Sobreiro, Renormalizability of the center-vortex free sector of YangMills theory, Phys. Rev. D 101, 085007 (2020).

[21] L. Del Debbio, M. Faber, J. Greensite, and S. Olejnik, Center dominance and $\mathrm{Z}(2)$ vortices in $\mathrm{SU}(2)$ lattice gauge theory, Phys. Rev. D 55, 2298 (1997).

[22] K. Langfeld, H. Reinhardt, and O. Tennert, Confinement and scaling of the vortex vacuum of SU(2) lattice gauge theory, Phys. Lett. B 419, 317 (1998).

[23] L. Del Debbio, M. Faber, J. Giedt, J. Greensite, and S. Olejnik, Detection of center vortices in the lattice YangMills vacuum, Phys. Rev. D 58, 094501 (1998). 
[24] M. Faber, J. Greensite, and S. Olejnik, Casimir scaling from center vortices: Towards an understanding of the adjoint string tension, Phys. Rev. D 57, 2603 (1998).

[25] P. de Forcrand and M. D'Elia, On the Relevance of Center Vortices to QCD, Phys. Rev. Lett. 82, 4582 (1999).

[26] J. Ambjorn, J. Giedt, and J. Greensite, Vortex structure versus monopole dominance in Abelian projected gauge theory, J. High Energy Phys. 02 (2000) 033.

[27] M. Engelhardt, K. Langfeld, H. Reinhardt, and O. Tennert, Deconfinement in SU(2) Yang-Mills theory as a center vortex percolation transition, Phys. Rev. D 61, 054504 (2000).

[28] M. Engelhardt and H. Reinhardt, Center projection vortices in continuum Yang-Mills theory, Nucl. Phys. B567, 249 (2000).

[29] R. Bertle, M. Engelhardt, and M. Faber, Topological susceptibility of Yang-Mills center projection vortices, Phys. Rev. D 64, 074504 (2001).

[30] H. Reinhardt, Topology of center vortices, Nucl. Phys. B628, 133 (2002).

[31] J. Gattnar, C. Gattringer, K. Langfeld, H. Reinhardt, A. Schafer, S. Solbrig, and T. Tok, Center vortices and Dirac eigenmodes in SU(2) lattice gauge theory, Nucl. Phys. B716, 105 (2005).

[32] L. E. Oxman, 4d ensembles of percolating center vortices and monopole defects: The emergence of flux tubes with $\mathrm{N}$-ality and gluon confinement, Phys. Rev. D 98, 036018 (2018).

[33] J. D. Länge, M. Engelhardt, and H. Reinhardt, Energy density of vortices in the Schrödinger picture, Phys. Rev. D 68, 025001 (2003).

[34] Daniel Zwanziger, Nonperturbative modification of the Faddeev-Popov formula and banishment of the naive vacuum, Nucl. Phys. B209, 336 (1982).

[35] G. Dell'Antonio and D. Zwanziger, Every gauge orbit passes inside the Gribov horizon, Commun. Math. Phys. 138, 291 (1991).

[36] P. van Baal, More (thoughts on) Gribov copies, Nucl. Phys. B369, 259 (1992).

[37] Ph. de Forcrand, J. Hetrick, A. Nakamura, and M. Plewnia, Gauge fixing on the lattice, Nucl. Phys. B, Proc. Suppl. 20, 194 (1991).

[38] G. Dell'Antonio and D. Zwanziger, Every gauge orbit passes inside the Gribov horizon, Commun. Math. Phys. 291 (1991).
[39] J. Greensite, S. Olejník, and D. Zwanziger, Center vortices and the Gribov horizon, J. High Energy Phys. 05 (2005) 038 .

[40] A. Maas, Instantons, monopoles, vortices, and the FaddeevPopov operator eigenspectrum, Nucl. Phys. A790, 566c (2007).

[41] H. Reinhardt and T. Tok, Abelian and center gauge fixing in continuum Yang-Mills theory for general gauge groups, Phys. Lett. B 500, 173 (2001).

[42] J. C. Vink and U.-J. Wiese, Gauge fixing on the lattice without ambiguity, Phys. Lett. B 289, 122 (1992).

[43] C. Ford, U. G. Mitreuter, T. Tok, A. Wipf, and J. M. Pawlowski, Monopoles, polyakov loops, and gauge fixing on the torus, Ann. Phys. (N.Y.) 269, 26 (1998).

[44] P. W. Higgs, Broken Symmetries and the Masses of Gauge Bosons, Phys. Rev. Lett. 13, 508 (1964).

[45] H. J. de Vega, Fermions and vortex solutions in Abelian and non-Abelian gauge theories, Phys. Rev. D 18, 2932 (1978).

[46] L. E. Oxman, Confinement of quarks and valence gluons in SU(N) Yang-Mills-Higgs models, J. High Energy Phys. 03 (2013) 038.

[47] C. D. Fosco, D. R. Junior, and L.E. Oxman, Quantum effects due to a moving Dirichlet point, Phys. Rev. D 101, 065014 (2020).

[48] D. R. Junior, L. E. Oxman, and G. M. Simões, 3D YangMills confining properties from a non-Abelian ensemble perspective, J. High Energy Phys. 01 (2020) 180.

[49] L. E. Oxman and H. Reinhardt, Effective theory of the D = 3 center vortex ensemble, Eur. Phys. J. C 78, 177 (2018).

[50] L. E. Oxman and G. M. Simões, k-strings with exact Casimir law and Abelian-like profiles, Phys. Rev. D 99, 016011 (2019).

[51] A. Gorsky, M. Shifman, and A. Yung, Non-Abelian Meissner effect in Yang-Mills theories at weak coupling, Phys. Rev. D 71, 045010 (2005).

[52] A. Hanany and D. Tong, Vortices, instantons and branes, J. High Energy Phys. 03 (2003) 037.

[53] R. Auzzi, S. Bolognesi, J. Evslin, K. Konishi, and A. Yung, Nonabelian superconductors: Vortices and confinement in $N=2$ SQCD, Nucl. Phys. B673, 187 (2003).

[54] L. E. Oxman and D. Vercauteren, Exploring center strings in $S U(2)$ and $S U(3)$ relativistic Yang-Mills-Higgs models, Phys. Rev. D 95, 025001 (2017).

[55] A. Maas, On the spectrum of the Faddeev-Popov operator in topological background fields, Eur. Phys. J. C 48, 179 (2006). 